\title{
Verbal discrimination reversal in a whole/part re-pairing transfer paradigm*
}

\author{
WILLIAM P. WALLACE and TIMOTHY J. SAWYER \\ University of Nevada, Reno, Nevada 89507
}

\begin{abstract}
Whole/part transfer and re-pairing of rights and wrongs were employed in a study of verbal discrimination reversal learning. The whole/part and re-pairing procedures were used to create reversal conditions $(0 \%, 50 \%$, or $100 \%$ reversal) that were similar at the outset of reversal learning in that all correct alternatives on the reversal list were higher in situational frequency than their respective incorrect alternatives. Similarly, reversal lists were constructed that were similar in that all incorrect alternatives on the reversal list were higher in situational frequency than their respective correct alternatives. The results were consistent with the existing literature on verbal discrimination reversal, as performance was impaired when items reversed functions $(50 \%$ and $100 \%$ reversal). There was little evidence that uniform frequency relations between rights and wrongs affected ease of reversal learning.
\end{abstract}

The purpose of the present experiment was to investigate whether frequency discriminations were dominant in verbal discrimination reversal learning. Recent research and theorizing, while not entirely inconsistent with frequency theory (Ekstrand, Wallace, \& Underwood, 1966), have at least implied that acquisition of a reversal or partial reversal verbal discrimination list involves learning mechanisms other than frequency discrimination (e.g., Paul, 1968; Paul \& Callahan, 1972; Paul, Hoffman, \& Dick, 1970; Raskin, Boice, Rubel, \& Clark, 1968). The present experiment was initiated to determine if frequency units acquired in a prior verbal discrimination task influenced verbal discrimination reversal transfer.

The rationale for this experiment developed from an extension of studies on prefamiliarization training and verbal discrimination learning (VDL). It is known that familiarization training on items that are to serve subsequently as correct alternatives facilitates VDL relative to familiarization training on incorrect alternatives or both correct and incorrect alternatives (e.g., Lovelace, 1969; Underwood \& Freund, 1968; Wallace \& Nappe, 1970). In the present study, differential familiarization training was given to rights and wrongs in a VDL task so that Ss could transfer to VDL reversal conditions with either all the new rights greater than wrongs in situational frequency or all the wrongs greater than rights in situational frequency. For this purpose, three departures from standard VDL procedures were required. First, pairs were presented differing numbers of times prior to transfer; in the present case, 12 VDL pairs appeared eight times each and $12 \mathrm{VDL}$ pairs appeared two times each in the first stage of the experiment. Second, a whole/part transfer

*This research was supported by Grant MH 21895 from the National Institute of Mental Health. The authors are grateful to Michael D. Murphy for his assistance in the preparation of materials used in this experiment. Requests for reprints should be sent to William P. Wallace, Department of Psychology, University of Nevada, Reno, Nevada 89507 . task was employed. The first VDL list consisted of 24 pairs and only 12 pairs were involved in the second list. Third, re-pairing of first-list rights and wrongs was included in all conditions. Only one word from each of the 24 first-stage pairs was carried over into the second stage; thus, constructing second-stage VDL pairs required re-pairing first-stage rights and wrongs. It was always the case that an item from a pair that appeared eight times in the first list was paired with an item from a pair that appeared two times in the first list, and in such a way that the second list involved all the right items from pairs that appeared eight times paired with all the wrong items from pairs that appeared two times, or vice versa.

The critical advantage of this complex transfer procedure is that one may create lists such that all reversal conditions $(0 \%, 50 \%$, and $100 \%$ reversal) may start reversal learning either with the new correct alternatives higher in frequency value than their respective new incorrect alternatives or with the new incorrect alternatives higher in frequency value than the new correct alternatives. Normally, according to frequency theory, a $0 \%$ reversal group has rights greater than wrongs in frequency value by the critical point in acquisition ("reversal" transfer), whereas a 100\% reversal group has wrongs greater than rights at the start of reversal learning. A $50 \%$ reversal group has a mixture of both types of pairs.

The similarity between verbal discrimination reversal and studies on prefamiliarization training should be apparent. Prefamiliarization training on rights only (R) presumably produces frequency relations similar to those produced by initial training with a $0 \%$ reversal condition. Similarly, prefamiliarization training on wrongs only (W) may be likened to a $100 \%$ reversal condition, and prefamiliarization training on both rights and wrongs (B) may be analogous to $50 \%$ reversal. Reversal and familiarization operations have similar relative effects on VDL. Following familiarization treatments, $\mathrm{R}$ groups (Rs $>$ Ws) are superior to $\mathrm{W}$ groups 
(Ws $>$ Rs) and $\mathrm{W}$ groups are superior to $\mathrm{B}$ groups (mixed). In reversal acquisition, $0 \%$ reversal (Rs $>$ Ws) is superior to $100 \%$ reversal (Ws $>$ Rs), and $100 \%$ reversal is superior to 50\% reversal (mixed). The present experiment was addressed to the issue of whether the general right-wrong frequency relations were a major contributor to the ordering of different reversal transfer groups.

The present operations were designed to eliminate the "frequency-rule" differences among the various reversal conditions. For example, a $0 \%$ reversal group with all wrongs greater than rights in situational frequency and a $100 \%$ reversal group with all rights greater than wrongs could be included with the present procedures. In both cases, rights from first-list pairs presented two times would be re-paired with wrongs from first-list pairs presented eight times. Thus, at the start of the second list there would be a uniform eight to two advantage (in terms of number of prior exposures) favoring wrongs in a $0 \%$ reversal condition; however, there would be an eight to two advantage for the new rights in a $100 \%$ reversal condition.

Although the transfer paradigm described above may be rather complex, the logic of the experiment seems straightforward. New $0 \%, 50 \%$, and $100 \%$ reversal conditions are introduced that are atypical in the sense that a $0 \%$ reversal group has all Ws $>$ Rs at the start of reversal learning, a $100 \%$ reversal group has all Rs $>$ Ws at the start of reversal learning, and $50 \%$ groups do not have a mixture of both types of pairs. The basic question concerns whether the typical reversal findings (e.g., $0 \%$ reversal superior to $100 \%$ reversal) will be "reversed" with the present procedures (e.g., 100\% reversal superior to $0 \%$ reversal). That is, will the frequency rule relations override the reversal manipulation and influence reversal acquisition in a manner consistent with prefamiliarization studies (e.g., Lovelace, 1969)? In addition, "conventional" groups are included $(0 \%$, $\mathrm{Rs}>\mathrm{Ws}$ and $100 \%$, Ws $>\mathrm{Rs}$ ), which should reveal whether the novel procedures per se (the whole/part re-pairing transfer paradigm) produce atypical reversal results.

\section{METHOD}

\section{Materials}

The materials used to construct the verbal discrimination lists were low-frequency (Thorndike-Lorge values between four and six occurrences per million words) three- or four-letter words. Two different sets of 24 pairs were constructed. Each of these sets served as first-list materials for half of the Ss. Second lists for each of these sets of materials were derived by selecting one word from each of the 24 first-list pairs (12 rights and 12 wrongs) and re-pairing these words to form 12 new pairs.

During first-list learning, 12 pairs appeared eight times and 12 pairs appeared two times. Levels of the two variables in this study were determined by the specific items and new pairings that were used in the second list. The experiment involved a 3 by 2 factorial combination of percentage of rights and wrongs that reversed their function from the first list to the second list $(0 \%, 50 \%$, or $100 \%)$ and the situational frequency relations between rights and wrongs in the second list. (All second-list rights had eight exposures in the first list and the wrongs had two exposures or all second-list rights had two exposures in the first list and the wrongs had eight exposures.) The six major groups may be identified as follows: $0 \%-\mathrm{R}>\mathrm{W} ; 0 \%-\mathrm{W}>\mathrm{R}$; $50 \%-\mathrm{R}>\mathrm{W} ; 50 \%-\mathrm{W}>\mathrm{R} ; 100 \%-\mathrm{R}>\mathrm{W}$; and $100 \% \mathrm{~W}>\mathrm{R}$; where $0 \%, 50 \%$, and $100 \%$ refer to the percentage of items that reversed functions and $\mathrm{R}>\mathrm{W}$ refers to the case where at the outset of second-list acquisition the exposure frequency of the second-list rights exceeded the exposure frequency of the second-list wrongs and $\mathrm{W}>\mathrm{R}$ indicates the opposite.

There were two distinct second lists (appropriate to the two sets of materials), with half of the Ss learning each. Thus, all Ss learned essentially the same second list. Variations in specific right and wrong functions of items and the appropriate number of exposures for specific items were introduced in the first lists to create the different percent reversal and frequency groups. There were 12 different first lists, as a different first list was required for each of the two sets of materials and for each of the six major groups.

The following example involving a four-pair first list and a two-pair second list is provided to illustrate the experimental conditions and the principle of list construction. The following eight words were selected from one set of materials at random: gash, clog, epic, vat, cowl, doe, rig, and balk. The two second-list pairs selected from these words were gash-vat and clog-balk, with the italicized words the correct members of the pairs. The first list for Group 0\%-R > W included gash-epic, clog-doe, cowl-vat, and rig-balk, with the first two pairs presented eight times and the latter two pairs presented two times. The appropriate first list for Group $0 \% \mathrm{~W}>\mathrm{R}$ was identical to the preceding list, with the exception that gash-epic and clog-doe appeared two times and cowl-vat and rig-balk appeared eight times. The first lists for Group $100 \%-\mathrm{R}>\mathrm{W}$ and Group $100 \%-\mathrm{W}>\mathrm{R}$ were identical to Groups $0 \%-\mathrm{R}>\mathrm{W}$ and $0 \%-\mathrm{W}>\mathrm{R}$, respectively, with the exception that epic, doe, vat, and balk were the correct alternatives. The first lists for the $50 \%$ groups involved gash-epic, clog-doe, cowl-vat, and rig-balk. Thus, gash-vat was a nonreversed second-list pair and clog-balk was a reversed second-list pair. For Group 50\%-R $>$ W, gash-epic and clog-doe appeared eight times in the first list, and for Group 50\%-W > R, cowl-vat and rig-balk appeared eight times in the first list.

\section{Procedure}

For both lists, pairs were presented on a Stowe memory drum at a 2 -sec rate. List 1 was presented for one continuous study trial only. The 24 pairs were arranged in a single random order that required 120 sequential positions, since 12 pairs appeared eight times in the sequence and 12 pairs appeared twice each. The members of a pair appeared side by side with the correct alternative underlined. Correct words appeared equally often in left and right spatial positions. Although Ss were given standard verbal discrimination instructions prior to the first list, a test trial was not administered in an effort to avoid the additions of test trial frequencies.

The second list of 12 pairs was presented at a 2 -sec rate with the study-test method. On the test trials the pairs were presented with neither word underlined, and for each pair Ss pronounced the word they thought was correct. The Ss were completely informed about the re-pairing, the whole/part transfer, and the appropriate reversal condition prior to the start of second-list acquisition. The second list was practiced to a criterion of three successive perfect trials, and each $\mathrm{S}$ continued for a minimum of nine trials, regardless of whether the criterion was reached before the ninth trial. Three different random orders were used in presenting pairs during the second-list study and test trials.

\section{Subjects}

A total of 120 introductory psychology students participated in this experiment. There were six major groups with $20 \mathrm{Ss}$ in 
each. The Ss were run individually and assigned to groups at random, based on their order of appearance at the laboratory.

\section{RESULTS}

Since all Ss were given a minimum of nine "reversal" trials, the six major groups could be compared on total errors. Analyses of errors over the first nine trials and the first four trials (the most dramatic changes occurred over the first four trials) revealed the same statistical results; consequently, only the analysis on errors over the first four trials will be reported. The mean errors across the first four reversal trials are presented in Fig. 1. A 2 by 3 by 4 analysis of variance revealed a significant main effect for percent reversal $[F(2,114)=14.27$, $p<.01]$, a significant main effect for trials $[F(3,342)=$ $20.26, \mathrm{p}<.01]$, and a significant Percent Reversal by Trials interaction $[\mathrm{F}(6,342)=2.40, \mathrm{p}<.05]$. A "basement" effect may have contributed in part to this interaction, because the $0 \%$ reversal groups started out making very few errors and, hence, they demonstrated only a limited reduction in errors across trials.

Contrary to expectation, lists with all $R>W$ pairs were not easier to learn than lists with all $\mathrm{W}>\mathrm{R}$ pairs, although the main effect for frequency relation $[F(1,114)=3.66]$ and the Frequency Relation by Percent Reversal interaction $[F(2,114)=3.05]$ fell just short of statistical reliability. However, if there is an effect here, inspection of Fig. 1 suggests that the greater ease of learning an $\mathrm{R}>\mathrm{W}$ list compared to a $\mathrm{W}>\mathrm{R}$ list occurs only in the absence of reversing right and wrong functions. There was no evidence that a reversal or partial reversal list was easier to learn when the new rights were greater in prior exposure frequency than the new wrongs compared to when new wrongs were greater in frequency than new rights.

It should be noted that absolute within-pair frequency discriminability most likely differs between $\mathrm{R}>\mathrm{W}$ conditions and $\mathrm{W}>\mathrm{R}$ conditions, at least for $0 \%$ and $100 \%$ reversal. For example, if each right gained two frequency units per exposure and each wrong gained one frequency unit per exposure in the first list (see Ekstrand et al, 1966), then Group 0\%-R > W would have 16 to 2 frequency discriminations as the rights from first-list pairs presented eight times were re-paired with wrongs from first-list pairs presented twice. For Group $0 \% \mathrm{~W}>\mathrm{R}$ the frequency discriminations would be four to eight, since first-list rights presented twice were re-paired with first-list wrongs presented eight times. However, for the $100 \%$ reversal groups, the $\mathrm{R}>\mathrm{W}$ condition should present the more difficult list in terms of frequency discriminability. For $\mathrm{R}>\mathrm{W}$ the new rights were first-list wrongs presented eight times and the new wrongs were first-list rights presented two times, resulting in an eight to four frequency relation. For $W>R$ the new rights were first-list wrongs presented two times and the new wrongs were first-list rights presented eight times, resulting in a 2 to 16 frequency

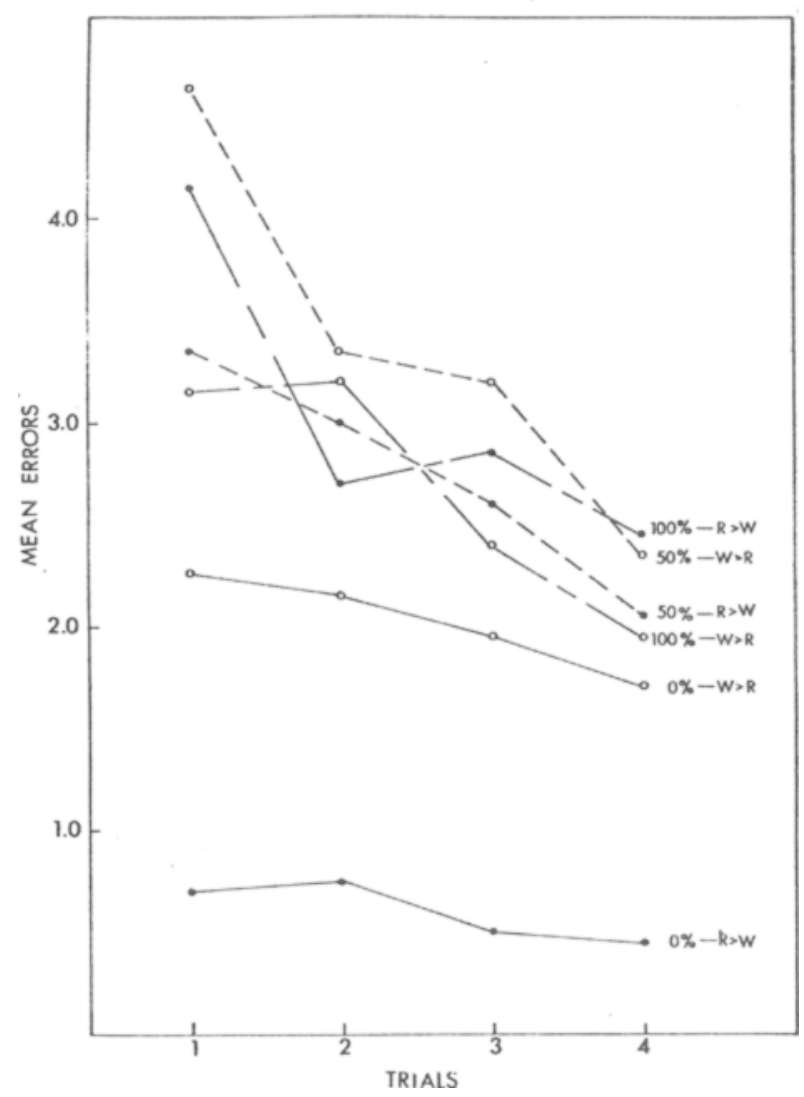

Fig. 1. Mean errors on the first four trials of verbal discrimination "reversal."

relation. Thus, for $100 \%$ reversal, pairs should be more discriminable under the $\mathrm{W}>\mathrm{R}$ rule than with the $\mathrm{R}>\mathrm{W}$ rule. The 50\% groups have both 16 to 2 and 8 to 4 frequency relations and do not differ in this regard.

In view of the above analysis, the statistical test on errors over the first four reversal trials was repeated, using only the data from the two $0 \%$ reversal groups and the two $100 \%$ reversal groups. The Percent Reversal by Frequency Relation interaction reached significance in this analysis of variance $[F(1,76)=5.74, p<.05]$. Thus, the possibility that early in reversal learning frequency discriminability influenced verbal discrimination reversal could not be dismissed.

The data from the $50 \%$ reversal groups were subjected to a 2 by 2 analysis of variance on total errors. For these groups one half of the pairs were reversed (equivalent to $100 \%$ reversal) and the other half were not reversed (equivalent to $0 \%$ reversal). Frequency relation was a between-gro'ıp variable, since for one $50 \%$ reversal group all rights were greater than wrongs in number of prior exposures and for the other $50 \%$ reversal group all wrongs were greater than rights in number of prior exposures. The results of the statistical analysis revealed a close parallel in errors over the first nine trials and the first four trials. For example, considering the first four trials, more errors were made on reversed pairs than on 


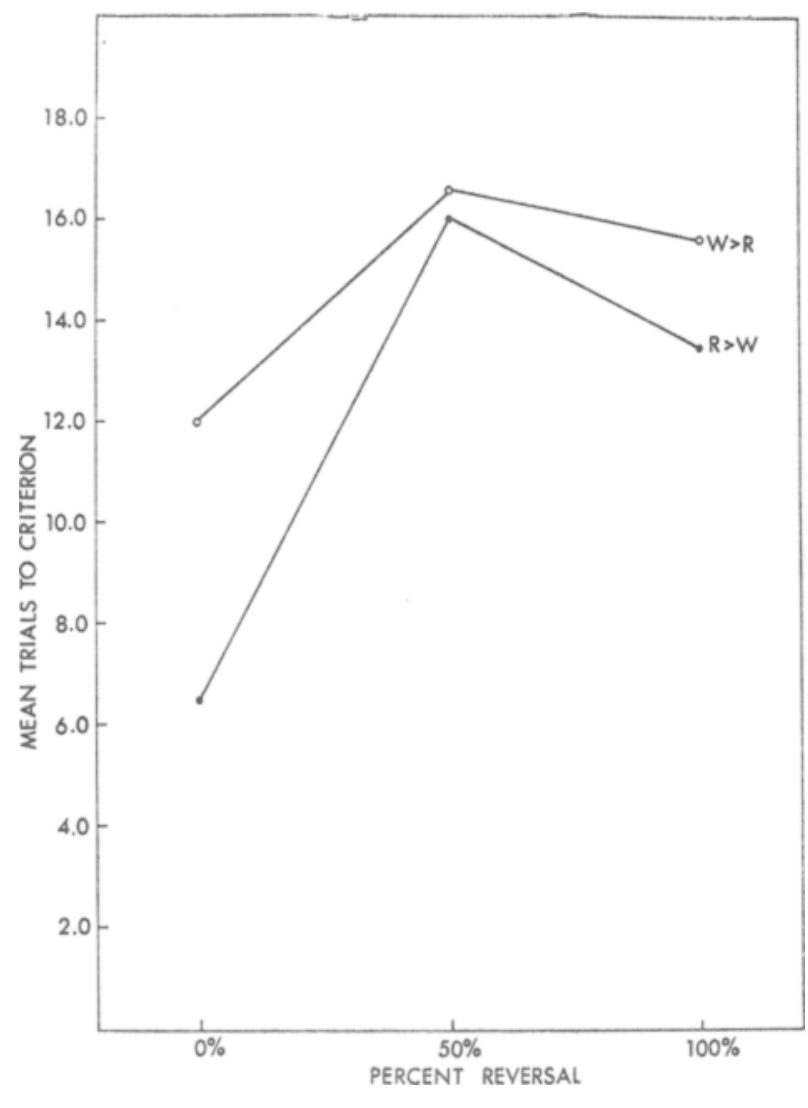

Fig. 2. Mean number of trials to three successive errorless trials.

nonreversed pairs $[F(1,38)=49.11, \mathrm{p}<.01]$, errors generally decreased across trials $[F(3,114)=14.97$, $\mathrm{p}<.01]$, and these two variables interacted $[F(3,114)=$ $7.11, p<.01]$. Frequency relation did not approach significance as a main effect or in interactions.

The groups were also compared on trials to criterion. A pattern of results similar to those reported on the errors measure was found with trials to criterion, which is depicted in Fig. 2. One problem that occurred with the trials to criterion measure was that the 12-pair transfer list proved difficult to learn. The experiment was discontinued if Ss did not reach the criterion of three successive perfect within 30 trials. Fourteen of the 120 Ss failed to reach criterion. These Ss were distributed across conditions as follows: $0 \%-\mathrm{R}>\mathrm{W}=1$, $0 \%-\mathrm{W}>\mathrm{R}=1,50 \% \mathrm{R}>\mathrm{W}=3,50 \% \mathrm{~W}>\mathrm{R}=4$, $100 \%-\mathrm{R}>\mathrm{W}=1,100 \% \cdot \mathrm{W}>\mathrm{R}=4$. In the statistical analysis, Ss who did not reach criterion were assigned a score of 33 (as if they would have achieved criterion in the minimum number of additional trials). It should be noted that in this experiment the reported effects are most likely underestimated. For example, two Ss in the $0 \%$ reversal groups received scores of 33 (when most likely they would have had higher scores), but in the more difficult $50 \%$ and $100 \%$ reversal conditions the scores for seven and five Ss, respectively, were assigned values of 33 and probably underestimated. Similarly, the error data indicated that numerically $R>W$ pairs were easier than $W>R$ pairs and, with the trials to criterion measure, the scores for five $S s$ in the $R>W$ conditions were underestimated, in contrast to the scores for nine Ss in the $\mathrm{W}>\mathrm{R}$ conditions.

A 2 by 3 analysis on trials to criterion indicated that percent reversal was significant $[\mathrm{F}(2,114)=7.36$, $p<.01]$. The main effect for the frequency relation fell short of significance $[F(1,114)=2.97]$, and the interaction $\mathrm{F}$ was less than 1.0.

The analysis was repeated, using trials to a criterion of one perfect in an effort to eliminate the necessity of assigning statistical scores. Still, a total of five Ss failed to achieve this criterion within the 30 trials $(0 \%-\mathrm{W}>\mathrm{R}=$ $1,50 \%-\mathrm{W}>\mathrm{R}=2,100 \%-\mathrm{R}>\mathrm{W}=1,100 \%-\mathrm{W}>\mathrm{R}=1$ ). The results of the trials to one perfect analysis paralleled those results obtained when the more stringent three perfect criterion was employed: Percent reversal was significant $[F(2,114)=6.22, p<.01]$, frequency relation was not significant $[F(1,114)=2.00]$, and the interaction $F$ was less than 1.0 .

\section{DISCUSSION}

The discussion may be brief. Departures in standard verbal discrimination procedures were introduced to manipulate frequency relations between rights and wrongs at the outset of reversal learning. Ss transferred to a second list with $0 \%, 50 \%$, and $100 \%$ of the first-list rights and wrongs reversed in function. However, for all conditions either each second-list right word had more first-list exposures than its corresponding wrong word or each second-list right had a lower exposure frequency than its corresponding wrong. It was reasoned that, if frequency discriminations were involved in verbal discrimination reversal, then for each reversal condition the new list with rights greater than wrongs should be easier than the wrongs greater than rights version. Clearly, this expectation was not confirmed by the data. With the possible exception of VDL that did not involve reversal $(0 \%$ reversal), the frequency relation did not affect verbal discrimination transfer. However, one complication with the present procedure must be noted. The $\mathrm{R}>\mathrm{W}$ case, compared to the $\mathrm{W}>\mathrm{R}$ case, may have presented frequency differences that were easier to detect for $0 \%$ reversal, but the opposite may have been true following $100 \%$ reversal. The Percent Reversal by Frequency Relation interaction was significant when the error analysis was restricted to the $0 \%$ and $100 \%$ reversal groups. Thus, the absence of the expected effects of frequency relations for the $100 \%$ reversal condition could indicate either of two possibilities: (1) Frequency discrimination does not dominate as the major mechanism for verbal discrimination reversal or (2) the present operations with $100 \%$ reversal resulted in more discriminable frequency differences for $W>R$ than $R>W$, whereas the opposite was true for $0 \%$ reversal. 
However, only the first possibility appears consistent with the results for the $50 \%$ reversal condition.

It should be noted that the present results are consistent with what is normally reported for verbal discrimination reversal: $100 \%$ reversal was more difficult than $0 \%$ reversal and $50 \%$ reversal was more difficult than $100 \%$ reversal. Certainly the procedural variations required for the present theoretical test (whole/part, re-pairing transfer) did not appear to produce atypical reversal effects. The problem for frequency theory is that the manipulated frequency relations did not produce atypical reversal effects either.

\section{REFERENCES}

Ekstrand, B. R., Wallace, W. P., \& Underwood, B. J. A frequency theory of verbal-discrimination learning. Psychological Review, 1966, 73, 566-578.

Lovelace, E. A. Verbal-discrimination learning: Varied familiarization on correct and incorrect items. Canadian Journal of Psychology, 1969, 23, 227-232.

Paul, C. Verbal discrimination reversal as a function of overlearning and percentage of items reversed: An extension. Journal of Verbal Learning \& Verbal Behavior, 1968, 7, 270-272.

Paul, C., \& Callahan, C. Effects of overtraining on verbal discrimination shifts: A differentiation-suppression hypothesis. Journal of Experimental Psychology, 1972, 92, 198-206.

Paul, C., Hoffman, C. D., \& Dick, S. Acquired (conditional) equivalence: $A$ basis for response-set effects in verbal-discrimination reversal performance. Journal of Experimental Psychology, 1970, 85, 361-367.

Raskin, D. C., Boice, C., Rubel, E. W., \& Clark, D. Transfer tests of the frequency theory of verbal discrimination learning. Journal of Experimental Psychology, 1968, 76, 521-529.

Underwood, B. J., \& Freund, J. S. Two tests of a theory of verbal-discrimination learning. Canadian Journal of Psychology, 1968, 22, 96-104.

Wallace, W. P., \& Nappe, G. W. Verbal-discrimination learning following a free-recall familiarization training procedure. Canadian Journal of Psychology, 1970, 24, 27-33.

(Received for publication August 6, 1973; revision received October 15,1973 ; accepted October 22, 1973.) 